PROCEEDINGS OF THE

AMERICAN MATHEMATICAL SOCIETY

Volume 139, Number 7, July 2011, Pages 2571-2576

S 0002-9939(2010)10676-7

Article electronically published on December 9, 2010

\title{
ALMOST QUARTER-PINCHED KÄHLER METRICS AND CHERN NUMBERS
}

\author{
MARTIN DERAUX AND HARISH SESHADRI
}

(Communicated by Richard A. Wentworth)

\begin{abstract}
Given $n \in \mathbb{Z}^{+}$and $\varepsilon>0$, we prove that there exists $\delta=\delta(\varepsilon, n)>0$ such that the following holds: If $\left(M^{n}, g\right)$ is a compact Kähler $n$-manifold whose sectional curvatures $K$ satisfy

$$
-1-\delta \leq K \leq-\frac{1}{4}
$$

and $c_{I}(M), c_{J}(M)$ are any two Chern numbers of $M$, then

$$
\left|\frac{c_{I}(M)}{c_{J}(M)}-\frac{c_{I}^{0}}{c_{J}^{0}}\right|<\varepsilon
$$

where $c_{I}^{0}, c_{J}^{0}$ are the corresponding characteristic numbers of a complex hyperbolic space form.

It follows that the Mostow-Siu surfaces and the threefolds of Deraux do not admit Kähler metrics with pinching close to $\frac{1}{4}$.
\end{abstract}

\section{INTRODUCTION}

It is well known that Riemannian manifolds of constant sectional curvature -1 must be locally isometric to real hyperbolic space. Allowing the sectional curvatures to be merely close to -1 (say with pinching close to 1 ; i.e. with a suitable normalization all sectional curvatures are between $-\alpha$ and -1 for some $\alpha$ close to $1)$, there are many more examples than hyperbolic manifolds.

In fact, in [3, Gromov and Thurston construct manifolds of dimension $\geq 4$ that admit negatively curved Riemannian metrics with pinching arbitrarily close to 1 but that do not admit any metric of constant curvature (in fact they do not admit any locally symmetric metric).

The Kähler analogue of the above examples is not well understood. Negatively curved Kähler metrics must have pinching at most $\frac{1}{4}$, as can be deduced from an inequality due to Berger (see [1]), and $\frac{1}{4}$-pinched metrics are precisely those locally isometric to complex hyperbolic space (of dimension $\geq 2$ ). It is not known, however, whether there exist negatively curved Kähler manifolds with pinching arbitrarily close to $\frac{1}{4}$ which are not biholomorphic to ball quotients.

The surfaces constructed by Mostow and Siu in [7] (as well as their threedimensional analogue; see [2]) admit Kähler metrics with negative sectional curvatures, but very little is known about the pinching of these metrics.

Received by the editors December 18, 2009 and, in revised form, June 28, 2010.

2010 Mathematics Subject Classification. Primary 53C21; Secondary 53C20.

(C)2010 American Mathematical Society 
The main result of this paper relates proximity to $\frac{1}{4}$-pinching with proximity of the ratios of Chern numbers to the corresponding ratios of Chern numbers of a complex hyperbolic manifold of the same dimension. This shows that neither the Mostow-Siu surfaces, nor the three-dimensional analogues constructed in [2] admit Kähler metric with pinching close to $\frac{1}{4}$. This answers a question raised by Pansu

in [8] (echoing a question of Gromov), which seems not to be answered anywhere in the literature.

\section{Pinching and Ratios of Chern numbers}

The main observation of this note is the following:

Theorem 2.1. Given $n \in \mathbb{Z}^{+}$and $\varepsilon>0$, there exists $\delta=\delta(\varepsilon, n)>0$ such that the following holds: If $\left(M^{n}, g\right)$ is a compact Kähler $n$-manifold whose sectional curvatures $K$ satisfy

$$
-1-\delta \leq K \leq-\frac{1}{4}
$$

and $c_{I}(M), c_{J}(M)$ are any two Chern numbers of $M$, then

$$
\left|\frac{c_{I}(M)}{c_{J}(M)}-\frac{c_{I}^{0}}{c_{J}^{0}}\right|<\varepsilon,
$$

where $c_{I}^{0}, \quad c_{J}^{0}$ are the corresponding characteristic numbers of a complexhyperbolic space-form.

Recall that Mostow and Siu constructed an infinite family of surfaces that admit negatively curved Kähler metrics, but the corresponding ratios $c_{1}^{2} / c_{2}$ do not approach 3 as one ranges through these examples (see the introduction of [7]). The ratios $c_{1}^{3} / c_{3}$ for the threefolds constructed in [2] certainly cannot approach 16 , since only a finite number of threefolds were constructed there.

Theorem 2.1 implies that the Mostow-Siu surfaces (as well as the threefolds in [2]) do not carry Kähler metrics with pinching close to $1 / 4$.

It is an interesting open question whether the conclusion of Theorem 2.1 holds if one assumes only the existence of almost quarter-pinched Riemannian metrics on a compact Kähler manifold. This question is natural in light of the result of Hernandez, Yau, Zheng ([4, [9]): If a compact Kähler manifold $M$ admits a negatively quarter-pinched Riemannian $g$ metric, then $(M, g)$ is holomorphically isometric to a complex hyperbolic space form.

\section{ProOF}

Let $V$ be a vector space over $\mathbb{R}$ of real dimension $2 n$ with an almost complex structure $J: V \rightarrow V$ and a $J$ invariant inner product $\langle$,$\rangle . Hence J^{2}=-I$ and $\langle J(v), J(w)\rangle=\langle v, w\rangle$ for all $v, w \in V$.

A curvature tensor on $V$ is a 4-linear map $R: V \times V \times V \times V \rightarrow \mathbb{R}$, i.e. an element of $\otimes^{4} V^{*}$, satisfying

(1) $R(X, Y, Z, W)=-R(Y, X, Z, W)=-R(X, Y, W, Z)$,

(2) $R(Z, W, X, Y)=R(X, Y, Z, W)$,

(3) $R(X, Y, Z, W)+R(X, W, Y, Z)+R(X, Z, W, Y)=0$.

If, in addition, $R$ satisfies

(4) $R(J(X), J(Y), Z, W)=R(X, Y, J(Z), J(W))=R(X, Y, Z, W)$,

then we say that $R$ is a Kähler curvature tensor. 
Let $\mathcal{R} \subset \bigotimes^{4} V^{*}$ denote the space of Kähler curvature tensors and $R_{0} \in \mathcal{R}$ denote the curvature tensor of the complex hyperbolic space $\mathbb{C} H^{n}$.

$R_{0}$ is given by

$$
\begin{aligned}
-4 R_{0}(u, v, z, w)= & \langle u, z\rangle\langle v, w\rangle-\langle u, w\rangle\langle v, z\rangle+\langle u, J(z)\rangle\langle v, J(w)\rangle \\
& -\langle u, J(w)\rangle\langle v, J(z)\rangle+2\langle u, J(v)\rangle\langle z, J(w)\rangle .
\end{aligned}
$$

It is well known that if $R$ is a Kähler curvature tensor on $V$ whose sectional curvatures are negative and $\frac{1}{4}$-pinched, then $R=R_{0}$. We generalize this in Proposition 3.2. Recall that we regard the space $\mathcal{R}$ of Kähler curvature tensors as a subspace of $\otimes^{4} V^{*}$. The inner product on $V$ canonically induces an inner product on $\otimes^{4} V^{*}$ and hence on $\mathcal{R}$.

In what follows, we will use the following notation: For arbitrary $u, v \in V$,

$$
K(u, v):=R(u, v, u, v) .
$$

If $u$ and $v$ are orthornormal, this is the sectional curvature of the 2-plane spanned by $u$ and $v$. For any $u$, let

$$
H(u):=R(u, J(u), u, J(u)) .
$$

If $u$ is a unit vector, this is the holomorphic sectional corresponding to $u$. Note that $H(u)=-1$ for all unit vectors $u$ if $R=R_{0}$.

Lemma 3.1. Given $\varepsilon>0$, there exists $\eta=\eta(\varepsilon)$ such that if $R$ is a Kähler curvature tensor with $|H(u)+1|<\eta$ for all unit vectors $u$, then $\left|R-R_{0}\right|<\varepsilon$.

Proof. Note that $\left|R-R_{0}\right|<\varepsilon$ if $\left|R(x, y, z, t)-R_{0}(x, y, z, t)\right|<\frac{\varepsilon}{(2 n)^{4}}$ for every orthonormal set $\{x, y, z, t\}$.

Let $u, v \in V$ be unit vectors. If $a, b$ are real numbers such that $a^{2}+b^{2}=1$, then (this calculation is from [5], Lemma 3 )

$$
\begin{gathered}
H(a u+b v)+H(a u-b v)=2 a^{4} H(u)+2 b^{4} H(v) \\
+12 a^{2} b^{2} R(u, J(u), v, J(v))-8 a^{2} b^{2} R(u, v, u, v) \\
H(a u+b J(v))+ \\
H(a u-b J(v))=2 a^{4} H(u)+2 b^{4} H(v) \\
+12 a^{2} b^{2} R(u, J(u), v, J(v))-8 a^{2} b^{2} R(u, J(v), u, J(v)) .
\end{gathered}
$$

We also have the equation

$$
R(u, v, u, v)+R(u, J(v), u, J(v))-R(u, J(u), v, J(v))=0,
$$

which follows from (3) and (4) in the definition of a Kähler curvature tensor. Take $a=b=\frac{1}{\sqrt{2}}$. It is clear that we can use these three equations to express $R(u, v, u, v)$ as a linear combination of

$$
H(u), H(v), H\left(\frac{u+v}{\sqrt{2}}\right), H\left(\frac{u-v}{\sqrt{2}}\right), H\left(\frac{u+J(v)}{\sqrt{2}}\right), H\left(\frac{u-J(v)}{\sqrt{2}}\right) .
$$

Moreover note that the coefficients in this expression are absolute constants, not dependent on anything.

On the other hand, one knows that the full curvature tensor can be expressed in terms of sectional curvatures. In fact [6],

$$
\begin{array}{r}
24 R(x, y, z, t)=K(x+z, y+t)+K(x-z, y-t)-K(x+z, y-t)-K(x-z, y+t) \\
-K(x+t, y+z)-K(x-t, y-z)+K(x+t, y-z)+K(x-t, y+z) .
\end{array}
$$


It is clear from this expression and the earlier one expressing $K(x, y)$ in terms of holomorphic sectional curvatures that $\left|R(x, y, z, t)-R_{0}(x, y, z, t)\right|<\frac{\varepsilon}{(2 n)^{4}}$ if $|H(u)+1|<\eta$ for an $\eta=\eta(\varepsilon, n)$.

Proposition 3.2. Given $\varepsilon>0$, there exists $\delta=\delta(\varepsilon, n)$ such that if the sectional curvatures of a Kähler curvature tensor $R \in \mathcal{R}$ satisfy

$$
-1-\delta \leq K \leq-\frac{1}{4}
$$

then

$$
\left|R-R_{0}\right|<\varepsilon
$$

in the norm on $\otimes^{4} V^{*}$.

Proof. By the lemma above, it is enough to show that there is a $\delta=\delta(\eta)>0$ such that $-1-\delta \leq K \leq-\frac{1}{4}$ implies $|H(u)+1|<\eta$ for all unit vectors $u$.

Let $u$ be any unit vector in $V$. Choose $v$ so that $\{u, J(u), v, J(v)\}$ is an orthonormal set. By (3) and (4) in the definition of a Kähler curvature tensor,

$$
K(u, v)+K(u, J(v))-R(u, J(u), v, J(v))=0 .
$$

We recall the following estimate of Berger (11, page 69, (7)). Note that although Berger works with positive pinching, his proof works for negative pinching as well. Also, our pinching hypothesis and the conclusion can be obtained from his by a rescaling. If $R$ is a curvature tensor (not necessarily Kähler) which is $\alpha$-pinched, i.e. $-\alpha \leq K \leq-\frac{1}{4}$, then

$$
|R(X, Y, Z, W)| \leq \frac{2}{3}\left(\alpha-\frac{1}{4}\right)
$$

for any orthonormal set $\{X, Y, Z, W\}$.

Let $\eta>0$ be as in Lemma 3.1. By Berger's estimate there exists $\delta_{1}=\delta_{1}(\eta)$ such that if the sectional curvatures $K$ satisfy

$$
-1-\delta_{1} \leq K \leq-\frac{1}{4}
$$

then

$$
|R(u, J(u), v, J(v))| \leq \frac{1}{2}+\frac{\eta}{6} .
$$

By (3.2) and (3.4)

$$
\begin{aligned}
K(u, v) & =-K(u, J(v))+R(u, J(u), v, J(v)) \\
& \geq \frac{1}{4}-\frac{1}{2}-\frac{\eta}{6}=-\frac{1}{4}-\frac{\eta}{6} .
\end{aligned}
$$

Of course, we have the same estimates for $K(u, J(v)), K(J(u), v))$ and for $K(J(u), J(v))$. Hence,

$$
-\frac{1}{4}-\frac{\eta}{6} \leq c \leq-\frac{1}{4}, \quad-\frac{1}{2}-\frac{\eta}{3}<R(u, J(u), v, J(v)) \leq-\frac{1}{2},
$$

where

$$
c \in\{K(u, v), K(u, J(v)), K(J(u), v), K(J(u), J(v))\} .
$$

Applying the second inequality in (3.6) to the orthonormal set

$$
\left\{\frac{1}{\sqrt{2}}(u-J(v)), \frac{1}{\sqrt{2}}(J(u)+v), \frac{1}{\sqrt{2}}(u+J(v)), \frac{1}{\sqrt{2}}(J(u)-v)\right\},
$$


we get

$$
R(u-J(v), J(u)+v, u+J(v), J(u)-v) \leq-2 .
$$

Expanding the left hand side,

$$
\begin{aligned}
& R(u-J(v), J(u)+v, u+J(v), J(u)-v)= \\
& H(u)+H(v)+2 R(u, J(u), v, J(v))-4 K(u, v) .
\end{aligned}
$$

Combining (3.6) and (3.7) we have

$$
H(u)+H(v)-4 K(u, v)<-1+\frac{2 \eta}{3} .
$$

(3.9) and the first inequality in (3.6) imply

$$
H(u)+H(v)<-2+\frac{2 \eta}{3} .
$$

Finally, using (3.3), the above inequality implies

$$
-1-\delta_{1}<H(u)<-1+\frac{2 \eta}{3}+\delta_{1} .
$$

Let

Then

$$
\delta=\min \left\{\frac{\eta}{3}, \delta_{1}\right\}
$$

$$
|H(u)+1|<\eta
$$

and the proof is complete since the unit vector $u$ was arbitrary.

Let $\omega$ denote the Kähler form of $V$, defined by $\omega(u, v)=\langle u, J(v)\rangle$. Then the volume form on $V$, with $V$ carrying the natural orientation coming from $J$, is just $\omega^{n}$.

Let $I=\left(a_{1}, \ldots, a_{n}\right)$ and $J=\left(b_{1}, \ldots, b_{n}\right)$ be multi-indices in $\left(\mathbb{Z}_{>0}\right)^{n}$ with $\Sigma_{i} i a_{i}=$ $\Sigma_{i} i b_{i}=n$. Given a Kähler curvature tensor $R$ we can form the corresponding Chern forms $c_{I}(R)=c_{1}^{a_{1}}(R) \wedge \ldots \wedge c_{n}^{a_{n}}(R)$ and $c_{J}(R)=c_{1}^{b_{1}}(R) \wedge \ldots \wedge c_{n}^{b_{n}}(R)$. These are elements of $\bigwedge^{n} V^{*}$.

Given $\varepsilon_{1}$ (to be chosen below), Proposition 3.2 (and the obvious continuous dependence of the Chern forms on the full curvature tensor) implies that there is a $\delta=\delta\left(\varepsilon_{1}, n\right)$ such that $-1-\delta<K<-1$ implies that

$$
\left|c_{I}(R)-c_{I}\left(R_{0}\right)\right|<\varepsilon_{1}, \quad\left|c_{J}(R)-c_{J}\left(R_{0}\right)\right|<\varepsilon_{1}
$$

in the norm on $\bigwedge^{n} V^{*}$.

Let $c_{I}\left(R_{0}\right)=a(n) \omega^{n}$ and $c_{J}\left(R_{0}\right)=b(n) \omega^{n}$. Choose $\varepsilon_{1}=\varepsilon_{1}(\varepsilon, n)$ so that

$$
\frac{a(n)-\varepsilon_{1}}{b(n)+\varepsilon_{1}}>\frac{a(n)}{b(n)}-\varepsilon, \quad \frac{a(n)+\varepsilon_{1}}{b(n)-\varepsilon_{1}}<\frac{a(n)}{b(n)}+\varepsilon .
$$

Now (3.10) implies

$$
\left(a(n)-\varepsilon_{1}\right) \omega^{n}<c_{I}(R)<\left(a(n)+\varepsilon_{1}\right) \omega^{n}
$$

and

$$
\left(b(n)-\varepsilon_{1}\right) \omega^{n}<c_{J}(R)<\left(b(n)+\varepsilon_{1}\right) \omega^{n} .
$$

We now turn to a compact Kähler manifold with almost-quarter pinching as in Theorem 2.1. We can integrate the above inequalities on $M$ to get

$$
\left(a(n)-\varepsilon_{1}\right) \operatorname{Vol}(M)<c_{I}(M)<\left(a(n)+\varepsilon_{1}\right) \operatorname{Vol}(M)
$$


and

$$
\left(b(n)-\varepsilon_{1}\right) \operatorname{Vol}(M)<c_{J}(M)<\left(b(n)+\varepsilon_{1}\right) \operatorname{Vol}(M) .
$$

Dividing these two inequalities and using (3.11) give the desired result.

\section{REFERENCES}

[1] M. Berger, Sur quelques variétés Riemanniennes suffisamment pincées, Bull. Soc. Math. France 88 (1960), 57-71. MR0133781 (24:A3606)

[2] M. Deraux, A negatively curved Kähler threefold not covered by the ball, Invent. Math. 160 (2005), 501-525. MR2178701 (2006j:32021)

[3] M. Gromov, W. P. Thurston, Pinching constants for hyperbolic manifolds, Invent. Math. 89 (1987), 1-12. MR892185 (88e:53058)

[4] L. Hernandez, Kähler manifolds and $\frac{1}{4}$-pinching, Duke Math. J. 62 (1991) 601-611. MR.1104810 (92b:53046)

[5] S. Kobayashi, S. I. Goldberg, Holomorphic bisectional curvature, J. Diff. Geom. 1 (1967), 225-233. MR0227901 (37:3485)

[6] S. Mendonca, D. Zhou, Expression of curvature tensors and some applications, Bol. Soc. Bras. Mat. 32 (2001), no. 2, 173-184. MR.1860868 (2002g:53048)

[7] G. D. Mostow, Y. T. Siu, A compact Kähler surface of negative curvature not covered by the ball, Ann. Math 112 (1980), 321-360. MR592294 (82f:53075)

[8] P. Pansu, Pincement des variétés à courbure négative d'après M. Gromov et W. Thurston, Séminaire de Théorie Spectrale et Géométrie, no. 4 (1985-1986), 101-113. MR.1046064 (91a:53067)

[9] S. T. Yau, F. Zheng, Negatively $\frac{1}{4}$-pinched Riemannian metric on a compact Kähler manifold, Invent. Math. 103 (1991), 527-535. MR1091617 (92a:53056)

Institut Fourier, Université de Grenoble I, 38402 Saint-Martin-D'Hères Cedex, FRANCE

E-mail address: deraux@ujf-grenoble.fr

Department of Mathematics, Indian Institute of Science, Bangalore 560012, India

E-mail address: harish@math.iisc.ernet.in 